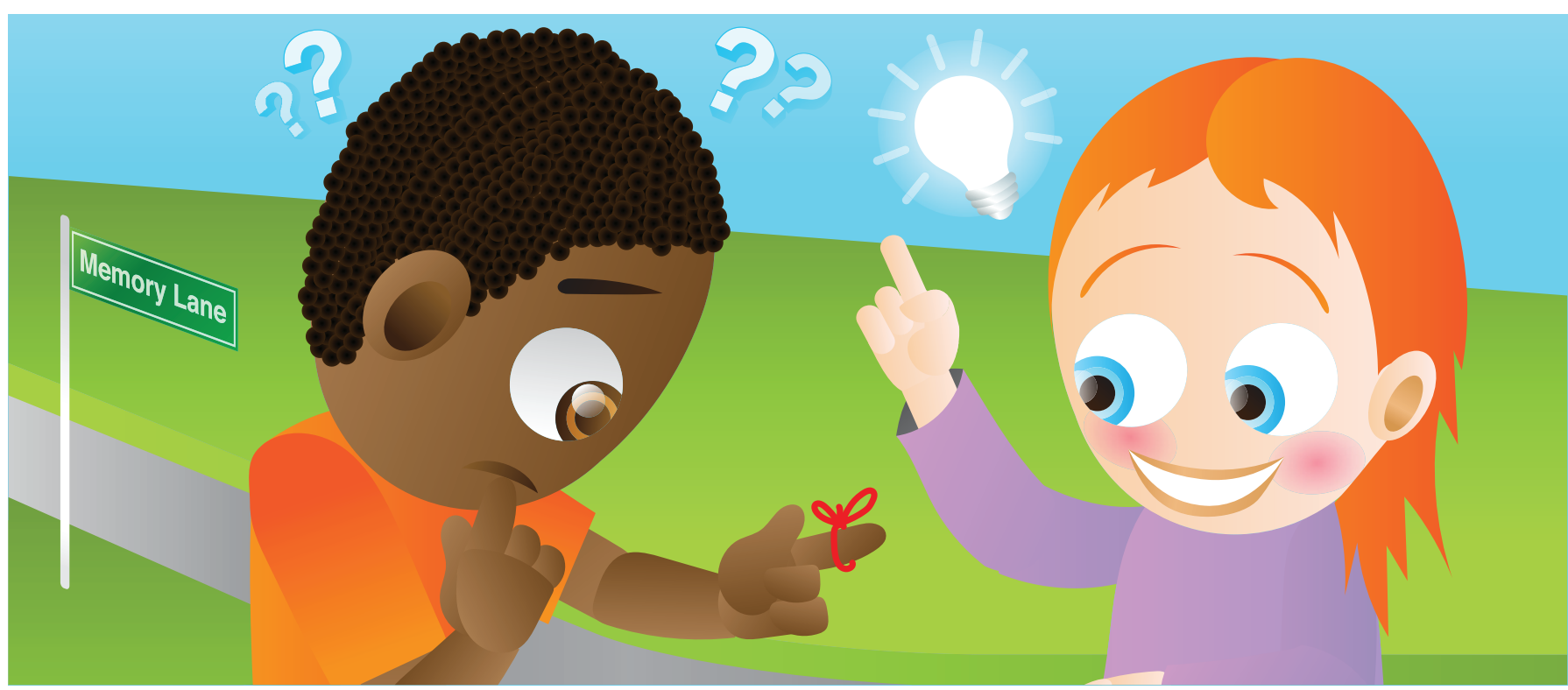

\title{
HOW BRAIN CELLS MAKE MEMORIES
}

\section{Elizabeth L. Johnson ${ }^{1,2 *}$ and Randolph F. Helfrich ${ }^{1 * *}$}

${ }^{1}$ Helen Wills Neuroscience Institute, University of California Berkeley, Berkeley, CA, USA, ${ }^{2}$ Department of Psychology, University of California Berkeley, Berkeley, CA, USA

\section{REVIEWED BY:}

$A B B Y$ 15 YEARS OLD
Remembering a lot of things at the same time is difficult. As an experiment, read these numbers: 07041776. Then, close your eyes and try to say them aloud, in order. How did you do? We would guess that you remembered around half of the numbers. Now, try again but think of the same numbers as a date: 07-04-1776. Did you remember more of the numbers this time? You just demonstrated something called working memory. Working memory ("WM" for short) is the ability to hold onto and process pieces of information. WM activates when you experience and remember events in your life, learn new facts, talk to people, read, and do math. WM is a basic human behavior. As shown in the numbers experiment, WM has limited capacity. How does the brain support WM? And, what is happening in the brain that limits our capacity to store multiple memories at the same time?

\section{HOW DOES THE BRAIN BUILD MEMORIES?}

Remembering is a complicated process. We will tell you about a few tricks that the human brain uses to remember a lot of things at the same time; but, first it is important to understand how memory works in the brain. The memory system is built into different parts of the brain. One key player is the part of 
WORKING

MEMORY

Is the ability to hold onto and process pieces of information.

your brain directly behind your forehead. This part is called the frontal lobe. When you think (or think about thinking!), you use your frontal lobe. Another key player in the memory system is buried deep inside your brain. This part is called the hippocampus, and it is very important for long-term memories, for instance, what you remember about how the brain builds memories tomorrow or another time in the future. For a picture of these brain structures and more information about the hippocampus, have a look at Ref. [1]. While looking at the brain structures tells us where memories are built, it does not tell us how memories are built. This article explains how brain cells build memories. We will explain why it is difficult to remember many things at the same time and then show you a few ways to improve your own memory.

To investigate the working memory (WM) system, we record electrical signals from people's brains while they hold onto and process pieces of information. We ask people to remember things, such as numbers, words, or pictures. Then, our electrical recordings show us what brain cells called "neurons" do when people remember things after a short time (usually between $1 \mathrm{~s}$ and $1 \mathrm{~min}$ ) [2]. When neurons are active, they deliver very small electric currents (much smaller than currents from wall sockets). These WM experiments show that the electric currents change depending on how much information you remember.

Normally, you have to remember a lot of things at once. For example, to understand this article, you have to remember what you just read while you are still reading. Maybe you're also thinking about what is for dinner, where you're having dinner, and when you have to be there. Remembering all of these different things depends on an electric current that cycle three to eight times per second [2-5]. This means that some neurons in the human brain fire together over and over again between three and eight times in $1 \mathrm{~s}$ [3].

We use computer tools to analyze different components of the brain signal. In Figure 1, we show you a picture of what the brain signal looks like and what the component for WM looks like. We also show you the component that is active when you process sensory information, such as the things you see or hear. You can see that sensory processing is much faster (between 30 and 100 cycles per second) than the WM component. Our brains have a trick to make memories out of this very fast sensory activity. The brain uses the slower, three to eight cycle waves from the WM system to group the faster sensory activity together [4]. Figure 1 shows that you can have, for example, seven faster cycles of sensory information within one WM cycle. This is a very effective trick for organizing seven pieces of information in WM. This trick also explains why it is hard to remember more than seven things at the same time. The speed of the WM system seems to be limited to three to eight cycles per second; that is, the same seven things cycle over and over again three to eight times per second in WM. This limitation seems to restrict the number of items that we can remember at the same time. 


\section{FIGURE}

Recording from a human brain: the brain signal (top) looks noisy and random. We can break down the signal into different components, which have different functions. For example, electric currents that cycle three to eight times per second are involved in memory function. Much faster currents (30 or more cycles per second) represent sensory information, such as the things you see or hear. So, the brain signal shown at the top seems to be a combination of different currents, allowing our brains to do a lot of things simultaneously. As shown in the gray-shaded area, one cycle of the slow WM activity can include, for example, seven cycles of the sensory information.

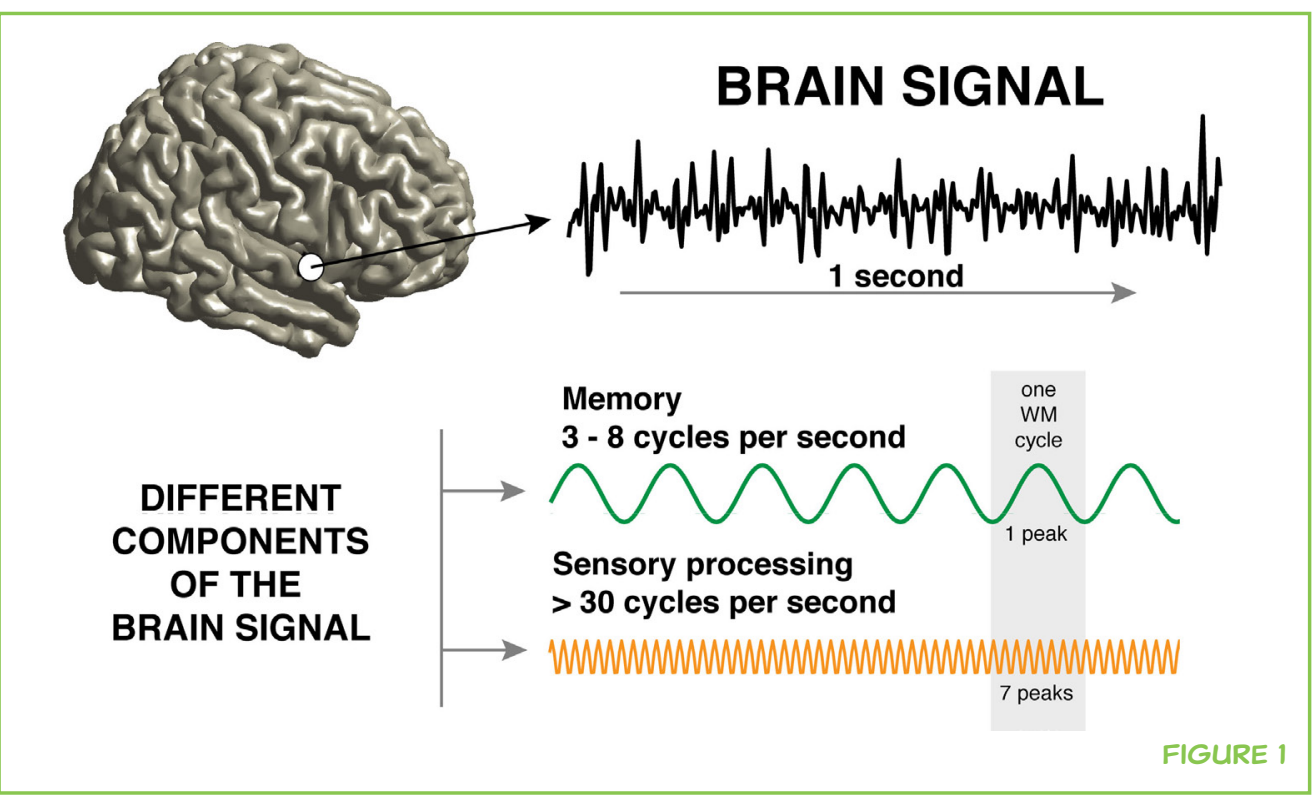

It should come as no surprise that the brain's WM function, which helps you think, read, and do math, is complicated. One reason for WM is so complicated is that the memory system is built into different parts of the brain, and these different parts of the brain need to talk to each other. Different parts of the brain talk to each other when neurons fire at the same time. To understand what this means, think about how several different people play instruments together in a band. The tune sounds good when the rhythms are coordinated, which means they go together in time. In the brain, we can see the memory rhythm cycling in different parts of the brain at the same time [2].

Another reason for WM is complicated is that the electrical signals in any one part of the brain are already very complex. As we showed you in Figure 1, researchers have to break down the brain signal in order to make sense of it. The brain signal is so complicated, because the brain does a lot of things at the same time. Our brains do these different things at different speeds, a phenomenon called "multiplexing." Think about a song being played with different instruments together. By breaking down these sounds, we are able to differentiate the guitar's rhythm from rhythms made by the saxophone or drums. It is similar with the brain signal, where we use computer analysis to separate the memory rhythm (the slow component) from other rhythms, such as the sensory processing rhythm (the faster component).

\section{WHY IS OUR ABILITY TO REMEMBER LIMITED?}

Think back to the WM experiment where we asked you to memorize the numbers 07041776 in order, and then to try again with the date 07-04-1776. Notice that it was easier to remember the numbers as a date, or set of three pieces of information, than it was to remember all eight numbers in order. 


\section{FIGURE 2}

Chunking information in one WM cycle: let us say you have to remember a set of different colors in the right order.

Holding onto two colors in your memory is simple. It gets more complicated if you have to remember six colors, especially if one color (here, red) appears twice. As you can see, the order becomes more important with more information to hold in memory. It probably helps if we tell you that all the colors appear in the American and German flags, in that order. That is, it probably helps if we show you a trick to put these six colors into two "chunks," making the colors easier to remember. The brain uses another trick to organize multiple pieces of information in three to eight cycles of electrical activity per second. First, different pieces of information are put into different time slots within one memory cycle. Then, our brains can automatically use timing to group different pieces of information together into chunks. When we chunk a lot of information into fewer, more meaningful pieces of information (such as two-colored flags versus six colors), we are helping our brains use their own organizational tricks!
And, have you noticed that $07-04-1776$ is another way to write July 4 th, 1776, the day the United States declared independence? You will probably have no trouble remembering this one piece of information. We show you more examples in Box 1.

\section{BOX 1 - WHAT IS ONE PIECE OF INFORMATION?}

Remembering a lot of different things at the same time is difficult, especially if something does not have a meaning to you. We asked you to remember the following eight numbers in order: 07041776. Then, we asked you to think about the numbers as a set of three pieces of information in a date: 07-04-1776. Finally, we told you that was another way of writing the single piece of information: July 4th, 1776, the day the United States declared independence. All of a sudden, eight meaningless numbers became one meaningful piece of information and that was easier to remember. If you did not remember the eight numbers in order, but you could remember Independence Day, then you successfully "chunked," or grouped together, the information in your memory.

Here are two more examples:

1. Let us say you meet five new friends at school and you have to remember their names: Daniel, Emily, Colin, Anna, and Bob. Try to recall their names in $1 \mathrm{~min}$.

How did you do? Maybe you forgot one or two! Well, what if we tell you that your five new friends' names start with the first five letters of the alphabet? $A \ldots B \ldots C \ldots$

2. Try to remember random colors; let us say: red, white, blue, black, red, and yellow. Again, that is difficult, but if you know that the first three colors are in the American flag and the last three colors are in the German flag, it might be easier. We show you how your brain might fit this information into a WM cycle in Figure 2.

2 items

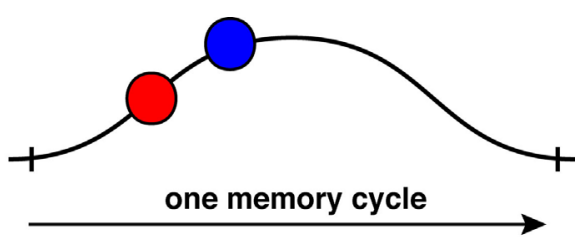

6 items

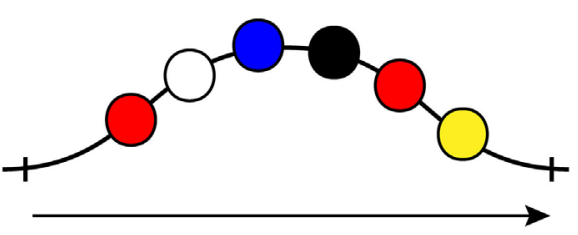

\section{2 items}

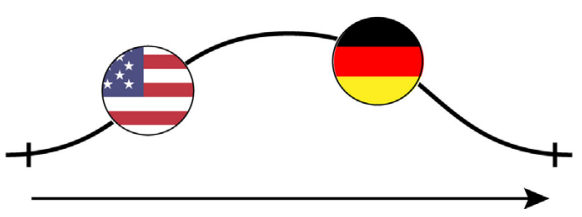

FIGURE 2

We have long understood that WM capacity is limited to approximately five to seven pieces of information [4]. As shown in Box 1, a piece of information can refer to something relatively meaningless like a single number or a color, or something more meaningful like a date or a flag.

As seen in Figure 1 (gray-shaded area), approximately seven cycles of sensory information fit into one WM cycle. To understand what this means, consider how we represent pieces of information in the brain. Think about Box 1 
(Figure 2), where you used the sensory process of vision to study the colors and the colored flags. To memorize a list of numbers, you would use vision to read the numbers; alternatively, you would use hearing if someone were to read the numbers to you. Electrical activity that cycles 30 or more times per second is involved in representing brain activity during sensory processing [4]. So, if you try to remember more than seven pieces of information at the same time, your brain might process all of the sensory information, but you might not actually remember all of the information later. This may be because more than seven items exceeds the capacity of the slower memory component that is, the WM cycle [4].

Now, let us do the math. If faster electrical currents cycle an average of 30 times in a second and slower electrical currents cycle an average of 5 times in a second, how many faster electrical cycles fit into each slower electrical cycle $(30 \div 5)$ ? The answer is 6 , which is consistent with our limited WM capacity of five to seven pieces of information. The number of times faster electrical currents fit into the slower WM electrical cycles might actually determine our WM capacity limits [4]! Have another look at Figure 1 for a picture of how the number of pieces of information (represented in electrical activity that cycles 30 or more times per second) can fit into a memory cycle (three to eight cycles per second). This relationship between fast- and slow-cycling electrical activities is important to how neurons make memories. Depending on how much information needs to be processed, the brain can speed up or slow down the slower, WM wave within the range of three to eight repeating cycle per second. Therefore, this slow rhythm can adapt, which helps the brain group the fast rhythm into meaningful pieces of information.

Thinking again to Box 1 (Figure 2), it may be easier to remember two-colored flags than to remember six colors in order, but it is more complicated to remember two flags of three colors each than it is to remember two single colors. Scientists are currently doing experiments to figure out how the brain supports WM for more complicated pieces of information - or, "chunks" of multiple pieces of information.

As mentioned above, the slower-cycling WM electrical activity is adaptive. This means that the WM cycle might slow down, from eight to three cycles per second, to incorporate more pieces of sensory information in one WM cycle [4]. Another way that the brain supports WM for chunked information is that the WM cycle organizes the sensory information in order based on timing [5]. In Figure 2, the first red-colored item occurs before the blue item, and the two red items occur at different times, separated in order by three other items. When we only have to remember two items, red and blue, the order of red-then-blue is simple. But, when we have to remember six items, the timing becomes more complicated - and important. This means that as we hold onto and process more and more pieces of information, the order in which different pieces of information enter the WM cycle becomes more and 
more important to WM function. The fast-cycling electrical activity, which represents pieces of sensory information and reflects the firing of neurons [2], actually occurs in ordered time slots within the slower WM cycle [5].

Putting it all together, electrical signals recorded from the human brain show us that we hold onto and process pieces of information in coordinated patterns of activity [2-5]. Neurons make memories by firing together in specific parts of the brain. That might be one mechanism for remembering multiple pieces of information at the same time. This complicated WM system allows us to make memories, and it may also be the reason why remembering a lot of things at the same time is so hard!

\section{IMPROVE YOUR MEMORY WITH SCIENCE}

Think back to Figure 2 again. In order to remember all six-colored items, we asked you to think about them as two-colored flags. As we described, this is called "chunking," in which you combine a lot of information into fewer, more manageable chunks. Chunking is a very effective strategy for remembering multiple things at the same time. As mentioned above, the brain uses the timing of the faster electrical waves to incorporate more and more information into each slower WM cycle. By chunking a lot of information into a single item or event, we are allowing our brains to handle more pieces of information. Usually, the brain automatically breaks incoming information into manageable pieces, making the information easier to process. You can also actively use chunking to improve your memory, for example, when you study to learn information.

You can associate and combine different pieces of information, however, you would like in order to make chunks. Your brain can actually use the timing of different cycles of electrical activity to make sense of relationships based on time, space, emotions, or anything else that holds meaning for you [6]. For example, think about two events that happened yesterday, such as talking to a friend and eating dinner. Which one happened first? Did they happen in different places? Did one make you laugh? Each of these questions adds meaning to the events, allowing you to chunk the events together in WM. So, make stories!

You may have guessed from our example WM experiments that the first author of this review is from the United States. Barring extensive brain damage, she will never forget the image of the American flag or the date July 4th, 1776, because it has particular meaning for her. What country do you think the second author is from? We showed you the American flag first and the German flag second (... yes, Germany!). It also helps to visualize your stories. 
Finally, because we hold onto pieces of information through cycles of repetitive electrical activity, memory improves with repetition. Make sure to tell your stories to help your brain hold onto your memories.

\section{REFERENCES}

1. Davachi, L., and Shohamy, D. 2014. Thanks for the memories.... Front. Young Minds 2:23. doi: 10.3389/frym.2014.00023

2. Johnson, E. L., and Knight, R. T. 2015. Intracranial recordings and human memory. Curr. Opin. Neurobiol. 31:18-25. doi: 10.1016/j.conb.2014.07.021

3. Rutishauser, U., Ross, I. B., Mamelak, A. N., and Schuman, E. M. 2010. Human memory strength is predicted by theta-frequency phase-locking of single neurons. Nature 464:903-7. doi: 10.1038/nature08860

4. Lisman, J. E., and Jensen, O. 2013. The theta-gamma neural code. Neuron 77:1002-16. doi: 10.1016/j.neuron.2013.03.007

5. Axmacher, N., Henseler, M. M., Jensen, O., Weinreich, I., Elger, C. E., and Fell, J. 2010. Cross-frequency coupling supports multi-item working memory in the human hippocampus. Proc. Natl. Acad. Sci. U.S.A. 107:3228-33. doi: 10.1073/ pnas.0911531107

6. Craik, F. I., and Lockhart, R. S. 1972. Levels of processing: a framework for memory research. J. Verbal Learn. Verbal Behav. 11(6):671-84. doi: 10.1016/S0022$5371(72) 80001-X$

SUBMITTED: 30 September 2015; ACCEPTED: 05 April 2016; PUBLISHED ONLINE: 28 April 2016.

EDITED BY: Robert T Knight, University of California, Berkeley, USA

CITATION: Johnson EL and Helfrich RF (2016) How Brain Cells Make Memories. Front. Young Minds 4:5. doi:10.3389/frym.2016.00005

CONFLICT OF INTEREST STATEMENT: The authors declare that the research was conducted in the absence of any commercial or financial relationships that could be construed as a potential conflict of interest.

COPYRIGHT @ 2016 Johnson and Helfrich. This is an open-access article distributed under the terms of the Creative Commons Attribution License (CC BY). The use, distribution or reproduction in other forums is permitted, provided the original author(s) or licensor are credited and that the original publication in this journal is cited, in accordance with accepted academic practice. No use, distribution or reproduction is permitted which does not comply with these terms. 


\section{REVIEWED BY}

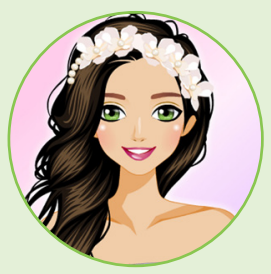

\section{ABBY, 15 YEARS OLD}

I am a freshman in high school. My favorite classes are physics and history because I love figuring out why things are the way they are. That fascination began when I was little and my mom, who is a neurosurgeon, set up experiments for me to do in her lab. When I am not busy with school, I spend my free time riding my horse and hanging out with my friends.

\section{AUTHORS}

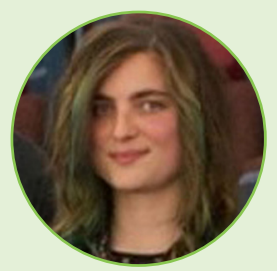

\section{ELIZABETH L. JOHNSON}

I find it incredible that this organ in our heads can make us so smart and so human. I want to understand memory, which allows us to think about so much information and put it all together, and craft identities based on personal experience. When I am not thinking about thinking, I like to explore weird and beautiful places, and share experiences with weird and beautiful people. *eljohnson@berkeley.edu

\section{RANDOLPH F. HELFRICH}

I am a medical doctor and a neuroscientist. For me, it is fascinating how the interactions of billions of cells in our brains give rise to cognition and behavior. Hence, I want to understand how neurons communicate and why the human brain is so special. When I am not in the lab, I enjoy traveling and playing the guitar or piano. **rhelfrich@berkeley.edu 